\title{
Two-dimensional digital demodulation for optical microscopes with spatial modulation of illumination
}

\author{
Volodymyr Borovytsky \\ Information Software Systems Ltd., \\ 15, Bozhenko str., Kyiv 03680, Ukraine, \\ e-mail: Volodymyr_Borovytsky@iss.org.ua
}

\begin{abstract}
It is proposed the new technique for the digital demodulation of images with two-dimensional spatial modulation of illumination. This technique is applicable for low contrast modulation with any phases of modulation that are different. Efficiency of the technique is demonstrated using images of test-objects formed by an optical microscope with and without spatial modulation of illumination.
\end{abstract}

Keywords: optical microscopy, spatial modulation of illumination, spatial resolution, digital demodulation, spatial harmonics.

Manuscript received 13.10.09; accepted for publication 22.10.09; published online 30.12.09.

The structural illumination microscopy or microscopy with spatial modulation of illumination (SMI) is a perspective branch of optical microscopy of high resolution [1-3]. Its principal advantage is a possibility to overcome the limit of spatial resolution caused by diffraction of optical waves on an aperture of microscope optics (MO) [1]. That is why the leading producers of optical microscopes such as Carl Zeiss and others have announced the new products based on implementation of SMI [2, 3].

The key idea of SMI microscopy is to apply nonuniform harmonic illumination [2-8]. As a result, the spatial harmonics of high frequencies located outside the MO bandwidth are shifted into it due to amplitude modulation of illumination on specimen surface and passed through a MO $[4,5]$. It is possible to extract these harmonics from the digital images with SMI captured by a digital camera and to put them on their place outside the bandwidth. This operation called the digital demodulation is performed using computers [4-7]. This demodulation makes the bandwidth of an imaging channel of an optical microscope wider, and it increases ability to resolve objects of small size called spatial resolution $[4,8]$.

Demodulation is the sophisticated operation: the spatial harmonics of high frequencies shifted into the MO bandwidth drop on the harmonics located inside the MO bandwidth. The separation of these harmonics is getting more complicated in two-dimensional case when spatial harmonics overlay in two dimensional spatial frequency space. In $[4,5]$ this separation is considered for the partial case when SMI phases are equal to 0 , $+\pi / 2,-\pi / 2$. In [6] separation is made using iterative procedures that requires many calculations with numerous digital images with SMI. In $[7,8]$ three onedimensional demodulations are applied for a set of the digital images with one directional SMI to form the twodimensional result. Two-dimensional demodulation still remains a complicated problem due to absence of a direct analytical solution for separation of the spatial harmonics. The goal of this work is to find a solution of this problem.

Let us consider the typical demodulation of images with two-dimensional SMI. To perform demodulation, it is necessary to capture five images in which SMI pattern has relative phases $(0,0),\left(\Delta \varphi_{01}, 0\right),\left(\Delta \varphi_{02}, 0\right),\left(0, \Delta \varphi_{01}\right)$, $\left(0, \Delta \varphi_{02}\right)$. Then, it has to make two-dimensional fast Fourier transform of each image and to get five corresponding complex spatial spectra $[4,5]$. Each spectrum contains five components, and it is necessary to separate them $[4,5]$. Four components are shifted by the +/-SMI spatial frequency along the axes of twodimensional SMI [4].

Five spatial spectra with five components in each spectrum can be written as a system of five equations: 


$$
\left\{\begin{array}{l}
{\left[\begin{array}{l}
{\left[-0.5 \cdot k_{M}\right] \cdot U\left(v_{X}, v_{Y}\right)+0.125 \cdot k_{M} \cdot\left(U\left(v_{X}+v_{M}, v_{Y}\right)+U\left(v_{X}-v_{M}, v_{Y}\right)+\right.} \\
\left.+U\left(v_{X}, v_{Y}+v_{M}\right)+U\left(v_{X}, v_{Y}-v_{M}\right)\right)=U_{\varphi 00}\left(v_{X}, v_{Y}\right) \\
{\left[1-0.5 \cdot k_{M}\right] \cdot U\left(v_{X}, v_{Y}\right)+0.125 \cdot k_{M} \cdot\left(U\left(v_{X}+v_{M}, v_{Y}\right) \cdot \exp \left(j \cdot \Delta \varphi_{10}\right)+\right.} \\
\left.+U\left(v_{X}-v_{M}, v_{Y}\right) \cdot \exp \left(-j \cdot \Delta \varphi_{10}\right)+U\left(v_{X}, v_{Y}+v_{M}\right)+U\left(v_{X}, v_{Y}-v_{M}\right)\right)=U_{\varphi 10}\left(v_{X}, v_{Y}\right) \\
{\left[1-0.5 \cdot k_{M}\right] \cdot U\left(v_{X}, v_{Y}\right)+0.125 \cdot k_{M} \cdot\left(U\left(v_{X}+v_{M}, v_{Y}\right) \cdot \exp \left(j \cdot \Delta \varphi_{20}\right)+\right.} \\
\left.+U\left(v_{X}-v_{M}, v_{Y}\right) \cdot \exp \left(-j \cdot \Delta \varphi_{20}\right)+U\left(v_{X}, v_{Y}+v_{M}\right)+U\left(v_{X}, v_{Y}-v_{M}\right)\right)=U_{\varphi 20}\left(v_{X}, v_{Y}\right) \\
{\left[1-0.5 \cdot k_{M}\right] \cdot U\left(v_{X}, v_{Y}\right)+0.125 \cdot k_{M} \cdot\left(U\left(v_{X}+v_{M}, v_{Y}\right)+U\left(v_{X}-v_{M}, v_{Y}\right)+\right.} \\
\left.+U\left(v_{X}, v_{Y}+v_{M}\right) \cdot \exp \left(j \cdot \Delta \varphi_{01}\right)+U\left(v_{X}, v_{Y}-v_{M}\right) \cdot \exp \left(j \cdot \Delta \varphi_{01}\right)\right)=U_{\varphi 01}\left(v_{X}, v_{Y}\right) \\
{\left[1-0.5 \cdot k_{M}\right] \cdot U\left(v_{X}, v_{Y}\right)+0.125 \cdot k_{M} \cdot\left(U\left(v_{X}+v_{M}, v_{Y}\right)+U\left(v_{X}-v_{M}, v_{Y}\right)+\right.} \\
\left.+U\left(v_{X}, v_{Y}+v_{M}\right) \cdot \exp \left(j \cdot \Delta \varphi_{02}\right)+U\left(v_{X}, v_{Y}-v_{M}\right) \cdot \exp \left(j \cdot \Delta \varphi_{02}\right)\right)=U_{\varphi 01}\left(v_{X}, v_{Y}\right)
\end{array}\right.}
\end{array}\right.
$$

where $U\left(v_{\mathrm{X}}, v_{\mathrm{Y}}\right)-2 \mathrm{D}$ spatial spectrum of the image without SMI; $v_{\mathrm{X}}, v_{\mathrm{Y}}-$ spatial frequencies; $v_{\mathrm{M}}-$ spatial frequency of SMI that is supposed equal along axes $v_{\mathrm{X}}, v_{\mathrm{Y}} ; \Delta \varphi_{10}, \Delta \varphi_{20}$, $\Delta \varphi_{01}, \Delta \varphi_{02}$ - relative SMI phases in the digital images with SMI: $\Delta \varphi_{10}, \Delta \varphi_{20}$ are introduced by shift of the SMI pattern along axis $\mathrm{X}$, phases $\Delta \varphi_{01}, \Delta \varphi_{02}$ - by shift of the SMI pattern along axis $\mathrm{Y} ; \mathrm{U}_{\varphi 00}\left(v_{\mathrm{X}}, v_{\mathrm{Y}}\right), \mathrm{U}_{\varphi 10}\left(v_{\mathrm{X}}, v_{\mathrm{Y}}\right), \mathrm{U}_{\varphi 20}\left(v_{\mathrm{X}}, v_{\mathrm{Y}}\right)$, $\mathrm{U}_{\varphi 01}\left(v_{\mathrm{X}}, v_{\mathrm{Y}}\right), \mathrm{U}_{\varphi 02}\left(v_{\mathrm{X}}, v_{\mathrm{Y}}\right)$ - five spatial spectra of the digital images with SMI when the SMI pattern has relative phases $(0,0),\left(\Delta \varphi_{01}, 0\right),\left(\Delta \varphi_{02}, 0\right),\left(0, \Delta \varphi_{01}\right),\left(0, \Delta \varphi_{02}\right)$, relatively; $k_{M}-$ contrast of SMI in these images.

$\left|\begin{array}{ccc}K_{M 2} & 1 & 1 \\ K_{M 2} & \exp \left(j \cdot \Delta \varphi_{10}\right) & \exp \left(-j \cdot \Delta \varphi_{10}\right) \\ K_{M 2} & \exp \left(j \cdot \Delta \varphi_{20}\right) & \exp \left(-j \cdot \Delta \varphi_{20}\right)\end{array}\right| \cdot\left|\begin{array}{c}U_{Y}\left(v_{X}, v_{Y}\right) \\ U_{10}\left(v_{X}, v_{Y}\right) \\ U_{20}\left(v_{X}, v_{Y}\right)\end{array}\right|=M_{X} \cdot\left|\begin{array}{l}U_{Y}\left(v_{X}, v_{Y}\right) \\ U_{10}\left(v_{X}, v_{Y}\right) \\ U_{20}\left(v_{X}, v_{Y}\right)\end{array}\right|=\left|\begin{array}{l}U_{\varphi 00}\left(v_{X}, v_{Y}\right) \\ U_{\varphi 10}\left(v_{X}, v_{Y}\right) \\ U_{\varphi 20}\left(v_{X}, v_{Y}\right)\end{array}\right|$

$\left|\begin{array}{ccc}K_{M 2} & 1 & 1 \\ K_{M 2} & \exp \left(j \cdot \Delta \varphi_{01}\right) & \exp \left(-j \cdot \Delta \varphi_{01}\right) \\ K_{M 2} & \exp \left(j \cdot \Delta \varphi_{02}\right) & \exp \left(-j \cdot \Delta \varphi_{02}\right)\end{array}\right| \cdot\left|\begin{array}{l}U_{X}\left(v_{X}, v_{Y}\right) \\ U_{01}\left(v_{X}, v_{Y}\right) \\ U_{02}\left(v_{X}, v_{Y}\right)\end{array}\right|=M_{Y} \cdot\left|\begin{array}{l}U_{X}\left(v_{X}, v_{Y}\right) \\ U_{01}\left(v_{X}, v_{Y}\right) \\ U_{02}\left(v_{X}, v_{Y}\right)\end{array}\right|=\left|\begin{array}{l}U_{\varphi 00}\left(v_{X}, v_{Y}\right) \\ U_{\varphi 01}\left(v_{X}, v_{Y}\right) \\ U_{\varphi 02}\left(v_{X}, v_{Y}\right)\end{array}\right|$

where $\mathrm{U}_{00}\left(v_{\mathrm{X}}, v_{\mathrm{Y}}\right), \quad \mathrm{U}_{10}\left(v_{\mathrm{X}}, v_{\mathrm{Y}}\right), \quad \mathrm{U}_{20}\left(\mathrm{v}_{\mathrm{X}}, v_{\mathrm{Y}}\right), \mathrm{U}_{01}\left(v_{\mathrm{X}}, v_{\mathrm{Y}}\right)$, $\mathrm{U}_{02}\left(v_{\mathrm{X}}, v_{\mathrm{Y}}\right)$ - symbolic definition of the components $\mathrm{U}\left(v_{\mathrm{X}}, v_{\mathrm{Y}}\right), \mathrm{U}\left(\mathrm{v}_{\mathrm{X}}+v_{\mathrm{M}}, v_{\mathrm{Y}}\right), \mathrm{U}\left(\mathrm{v}_{\mathrm{X}}-\mathrm{v}_{\mathrm{M}}, v_{\mathrm{Y}}\right), \mathrm{U}\left(\mathrm{v}_{\mathrm{X}}, v_{\mathrm{Y}}+v_{\mathrm{M}}\right)$, $\mathrm{U}\left(v_{\mathrm{X}}, v_{\mathrm{Y}}-v_{\mathrm{M}}\right)$, relatively, they are introduced to simplify the formulas; $U_{Y}\left(v_{X}, v_{Y}\right), U_{X}\left(v_{X}, v_{Y}\right)$ - sums for the definite components of the spatial spectra (1), respectively:

$U_{Y}\left(\mathrm{v}_{X}, \mathrm{v}_{Y}\right)=K_{M 2} \cdot U_{00}\left(\mathrm{v}_{X}, \mathrm{v}_{Y}\right)$

$+U_{01}\left(v_{X}, v_{Y}\right)+U_{02}\left(v_{X}, v_{Y}\right)$

$U_{X}\left(v_{X}, v_{Y}\right)=K_{M 2} \cdot U_{00}\left(v_{X}, v_{Y}\right)+$

$U_{10}\left(v_{X}, v_{Y}\right)+U_{20}\left(v_{X}, v_{Y}\right)$

$M_{X}, M_{Y}$ - matrixes of the complex coefficients that describes the linear relationship between the components $\mathrm{U}\left(v_{\mathrm{X}}, v_{\mathrm{Y}}\right), \mathrm{U}\left(\mathrm{v}_{\mathrm{X}}+v_{\mathrm{M}}, v_{\mathrm{Y}}\right), \mathrm{U}\left(v_{\mathrm{X}}-v_{\mathrm{M}}, v_{\mathrm{Y}}\right), \mathrm{U}\left(v_{\mathrm{X}}, v_{\mathrm{Y}}+v_{\mathrm{M}}\right)$, $\mathrm{U}\left(v_{\mathrm{X}}, v_{\mathrm{Y}}-v_{\mathrm{M}}\right)$ and spatial spectra of five images with different SMI phases $\mathrm{U}_{\mathrm{Y}}\left(v_{\mathrm{X}}, v_{\mathrm{Y}}\right), \mathrm{U}_{\mathrm{X}}\left(\mathrm{v}_{\mathrm{X}}, v_{\mathrm{Y}}\right), \mathrm{U}_{\varphi 10}\left(v_{\mathrm{X}}, v_{\mathrm{Y}}\right)$, $\mathrm{U}_{\varphi 20}\left(v_{\mathrm{X}}, v_{\mathrm{Y}}\right), \mathrm{U}_{\varphi 01}\left(v_{\mathrm{X}}, v_{\mathrm{Y}}\right), \mathrm{U}_{\varphi 02}\left(\mathrm{v}_{\mathrm{X}}, \mathrm{v}_{\mathrm{Y}}\right)$, relatively; $\mathrm{K}_{\mathrm{M} 2}-$ coefficient introduced to take into account influence of the contrast $k_{M}$ of SMI in the digital images:
The system (1) is not a pure linear system of five equations. It has to point out that the relative SMI phases $\Delta \varphi_{10}, \Delta \varphi_{20}$ are defined along the axis of spatial coordinate $\mathrm{X}$, and $\Delta \varphi_{01}, \Delta \varphi_{02}$ - along the axis of spatial coordinate $\mathrm{Y}$. It is a principal difficulty in solving (1). To solve (1), it is proposed to group the first equation and two pair of two equations for SMI phase shift along the orthogonal axes of spatial coordinates X, Y. Now the system (1) can be rewritten as two linear systems of three equations in the following matrix form:
$K_{M 2}=\frac{\left[1-0.5 \cdot k_{M}\right]}{0.125 \cdot k_{M}}=8 \cdot \frac{\left[1-0.5 \cdot k_{M}\right]}{k_{M}}$

The solution of two systems (2) can be found analytically and written in the following form:

$$
\begin{aligned}
& \left|\begin{array}{l}
U_{X}\left(v_{X}, v_{Y}\right) \\
U_{10}\left(v_{X}, v_{Y}\right) \\
U_{20}\left(v_{X}, v_{Y}\right)
\end{array}\right|=M_{X}{ }^{-1} \cdot\left|\begin{array}{l}
U_{\varphi 00}\left(v_{X}, v_{Y}\right) \\
U_{\varphi 10}\left(v_{X}, v_{Y}\right) \\
U_{\varphi 20}\left(v_{X}, v_{Y}\right)
\end{array}\right| \\
& \left|\begin{array}{l}
U_{Y}\left(v_{X}, v_{Y}\right) \\
U_{01}\left(v_{X}, v_{Y}\right) \\
U_{02}\left(v_{X}, v_{Y}\right)
\end{array}\right|=M_{Y}{ }^{-1} \cdot\left|\begin{array}{l}
U_{\varphi 00}\left(v_{X}, v_{Y}\right) \\
U_{\varphi 01}\left(v_{X}, v_{Y}\right) \\
U_{\varphi 02}\left(v_{X}, v_{Y}\right)
\end{array}\right|
\end{aligned}
$$

where $M_{X}{ }^{-1}, M_{Y}^{-1}$ - inverse complex matrixes of the matrixes $M_{X}, M_{Y}(2)$, respectively.

These matrixes can be easy written in the following analytical form: 
$M_{X}{ }^{-1}=\frac{\left|\begin{array}{ccc}\frac{2 \cdot j}{K_{M 2}} \cdot \sin \left(\Delta \varphi_{10}-\Delta \varphi_{20}\right) & \frac{2 \cdot j}{K_{M 2}} \cdot \sin \left(\Delta \varphi_{20}\right) & -\frac{2 \cdot j}{K_{M 2}} \cdot \sin \left(\Delta \varphi_{10}\right) \\ \exp \left(-j \cdot \Delta \varphi_{10}\right)-\exp \left(-j \cdot \Delta \varphi_{20}\right) & \exp \left(-j \cdot \Delta \varphi_{20}\right)-1 & 1-\exp \left(-j \cdot \Delta \varphi_{10}\right) \\ \exp \left(j \cdot \Delta \varphi_{20}\right)-\exp \left(j \cdot \Delta \varphi_{10}\right) & 1-\exp \left(j \cdot \Delta \varphi_{20}\right) & \exp \left(j \cdot \Delta \varphi_{10}\right)-1 \\ 2 \cdot j \cdot\left(\sin \left(\Delta \varphi_{10}-\Delta \varphi_{20}\right)-\sin \left(\Delta \varphi_{20}\right)-\sin \left(\Delta \varphi_{10}\right)\right) & \frac{2 \cdot j}{K_{M 2} \cdot \sin \left(\Delta \varphi_{02}\right)}-\frac{2 \cdot j}{K_{M 2}} \cdot \sin \left(\Delta \varphi_{01}\right) \\ \frac{2 \cdot j}{K_{M 2}} \cdot \sin \left(\Delta \varphi_{01}-\Delta \varphi_{02}\right) & \exp \left(-j \cdot \Delta \varphi_{02}\right)-1 & 1-\exp \left(-j \cdot \Delta \varphi_{01}\right) \\ \exp \left(-j \cdot \Delta \varphi_{01}\right)-\exp \left(-j \cdot \Delta \varphi_{02}\right) & 1-\exp \left(j \cdot \Delta \varphi_{02}\right) & \exp \left(j \cdot \Delta \varphi_{01}\right)-1 \\ \exp \left(j \cdot \Delta \varphi_{02}\right)-\exp \left(j \cdot \Delta \varphi_{01}\right) & \end{array}\right|}{2 \cdot j \cdot\left(\sin \left(\Delta \varphi_{01}-\Delta \varphi_{02}\right)-\sin \left(\Delta \varphi_{02}\right)-\sin \left(\Delta \varphi_{01}\right)\right)}$

It is obvious that the unknown component $\mathrm{U}_{00}\left(v_{\mathrm{X}}, v_{\mathrm{Y}}\right)$ can be calculated using the sums $\mathrm{U}_{\mathrm{X}}\left(\mathrm{v}_{\mathrm{X}}, v_{\mathrm{Y}}\right)$, $\mathrm{U}_{\mathrm{Y}}\left(v_{\mathrm{X}}, \mathrm{v}_{\mathrm{Y}}\right)(2)$ :

$U_{00}\left(v_{X}, v_{Y}\right)=\left(U_{Y}\left(v_{X}, v_{Y}\right)-\right.$

$\left.U_{01}\left(v_{X}, v_{Y}\right)-U_{02}\left(v_{X}, v_{Y}\right)\right) / K_{M 2}=$ $=\left(U_{X}\left(v_{X}, v_{Y}\right)-\right.$

$\left.U_{10}\left(v_{X}, v_{Y}\right)-U_{20}\left(v_{X}, v_{Y}\right)\right) / K_{M 2}=$

As a result, all the components of the spatial spectrum can be calculated using the proposed analytical formulas (3). The components $\mathrm{U}_{10}\left(\mathrm{v}_{\mathrm{X}}, \mathrm{v}_{\mathrm{Y}}\right), \mathrm{U}_{20}\left(\mathrm{v}_{\mathrm{X}}, \mathrm{v}_{\mathrm{Y}}\right)$, $\mathrm{U}_{01}\left(v_{\mathrm{X}}, v_{\mathrm{Y}}\right), \mathrm{U}_{02}\left(v_{\mathrm{X}}, v_{\mathrm{Y}}\right)$ contain the pure spatial harmonics of high frequencies that has been located outside the MO bandwidth and shifted into it. It is possible to shift these harmonics back on their original location. To perform this operation, it has to shift the components $U_{10}\left(v_{X}, v_{Y}\right)$, $\mathrm{U}_{20}\left(v_{\mathrm{X}}, v_{\mathrm{Y}}\right), \mathrm{U}_{01}\left(v_{\mathrm{X}}, v_{\mathrm{Y}}\right), \mathrm{U}_{02}\left(\mathrm{v}_{\mathrm{X}}, v_{\mathrm{Y}}\right)$ to the definite zone of high spatial frequencies $[4,5]$ :

$$
U_{R}\left(v_{X}, v_{Y}\right)=\left\{\begin{array}{l}
U_{10}\left(v_{X}-v_{M}, v_{Y}\right),\left(\sqrt{v_{X}^{2}+v_{Y}^{2}}\right) \geq v_{R}, \\
v_{X}>0,\left|v_{X}\right| \leq\left|v_{Y}\right| U_{20}\left(v_{X}+v_{M}, v_{Y}\right), \\
\left(\sqrt{v_{X}^{2}+v_{Y}^{2}}\right) \geq v_{R}, v_{X}<0,\left|v_{X}\right| \leq\left|v_{Y}\right| \\
U_{0}\left(v_{X}, v_{Y}\right),\left(\sqrt{v_{X}^{2}+v_{Y}^{2}}\right) \leq v_{R} \\
U_{01}\left(v_{X}-v_{M}, v_{Y}\right),\left(\sqrt{v_{X}^{2}+v_{Y}^{2}}\right) \geq v_{R}, \\
v_{Y}>0,\left|v_{X}\right|>\left|v_{Y}\right| U_{02}\left(v_{X}+v_{M}, v_{Y}\right), \\
\left(\sqrt{v_{X}^{2}+v_{Y}^{2}}\right) \geq v_{R}, v_{Y}<0,\left|v_{X}\right|>\left|v_{Y}\right|
\end{array}\right.
$$

where $v_{R}$ is the radius of a circle in spatial frequency space, outside this circle it has to add the fragment of the restored components $\mathrm{U}_{10}\left(\mathrm{v}_{\mathrm{X}}, \mathrm{v}_{\mathrm{Y}}\right), \mathrm{U}_{20}\left(\mathrm{v}_{\mathrm{X}}, \mathrm{v}_{\mathrm{Y}}\right)$, $\mathrm{U}_{01}\left(v_{\mathrm{X}}, v_{\mathrm{Y}}\right), \mathrm{U}_{02}\left(v_{\mathrm{X}}, v_{\mathrm{Y}}\right)$; of course, $v_{\mathrm{R}}$ is not bigger than the MO bandwidth; $U_{R}\left(v_{X}, v_{Y}\right)$ - spatial spectrum of the high resolution digital image as a result of the digital demodulation. To get this image it is necessary to perform inverse two-dimensional fast Fourier transform of $U_{R}\left(v_{X}, v_{Y}\right)$ (4). It has to point out that SMI with the digital demodulation $(3,4)$ allows overcoming the diffraction limit of spatial resolution of MO:

1. At the beginning the spatial harmonics of high frequencies outside the MO bandwidth are shifted into it $[4,5]$.

2. These harmonics are extracted using the proposed mathematical apparatus (3).

3. These harmonics are shifted on their original location and added to the harmonics located inside the MO bandwidth (4). The final bandwidth of an imaging channel of an optical microscope is getting higher than the bandwidth of a diffraction-limited MO [9]. The increased bandwidth guarantees the higher spatial resolution as ability to resolve objects of small dimensions.

The computer simulation of an optical microscope with SMI proves the efficiency of the proposed technique $(3,4)$. It is selected the test objects as two dimensional structure of binary square bars (Fig. 1a). The spatial period of the bars is equal to $200 \mathrm{~nm}$ along axes $X, Y$. The MO has numerical aperture 0.95 and operation wavelength is $550 \mathrm{~nm}$. According to the Abbe formula, it has spatial resolution as the minimal resolved spatial period of a grating equal to $298 \mathrm{~nm}$ [9]. The spatial period of a grating is only 0.75 of the minimal 
resolved spatial period, and the bars cannot be resolved in the image formed by the MO even after digital deconvolution (see Figs $1 \mathrm{~b}$ and $1 \mathrm{c}$ ). It is illustrated in the space of spatial harmonics: the principal spatial frequency of the bars is higher than the MO bandwidth (see Figs 1d, e, f). a)

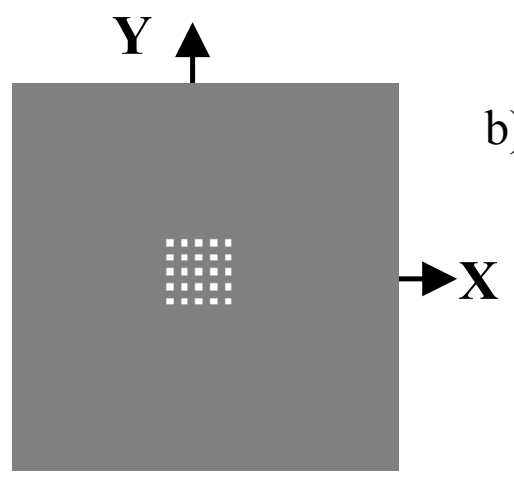

d)

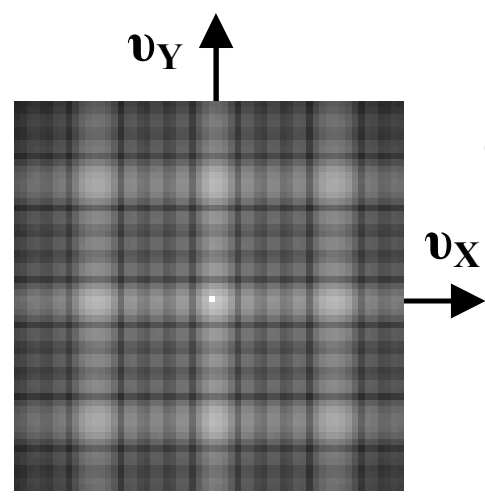

g)

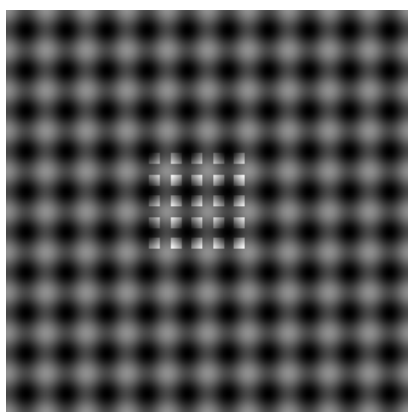

j)

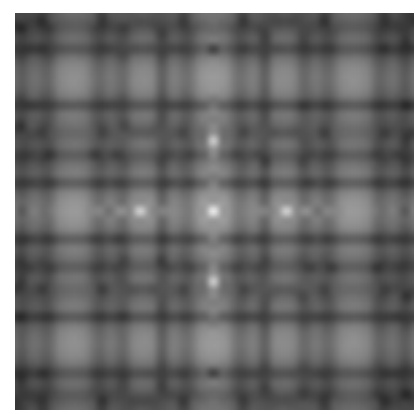

b)

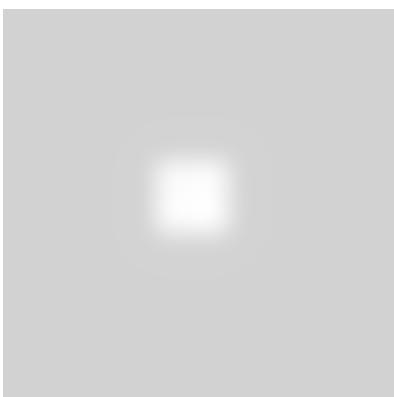

e)

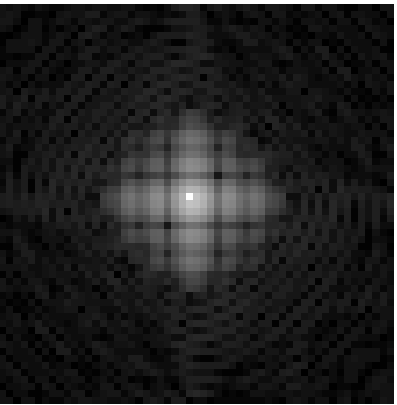

h)

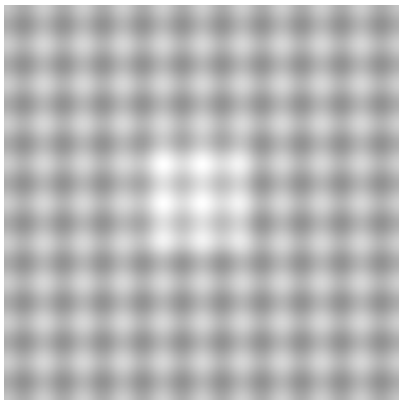

k)

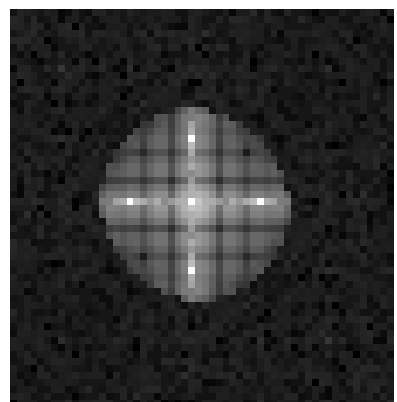

c)

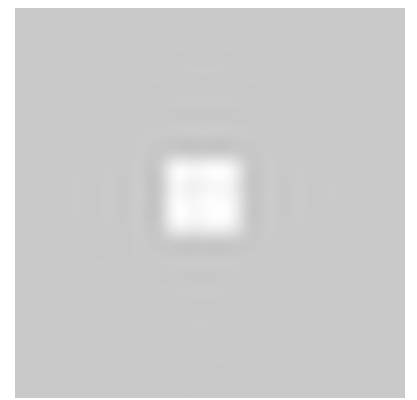

f)

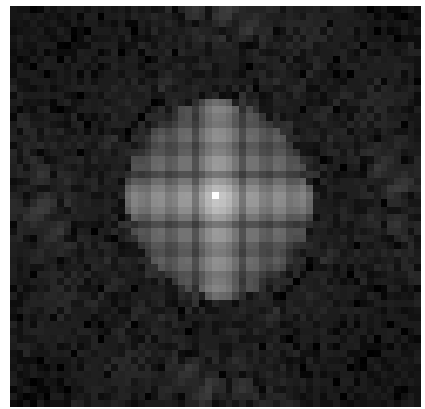

i)

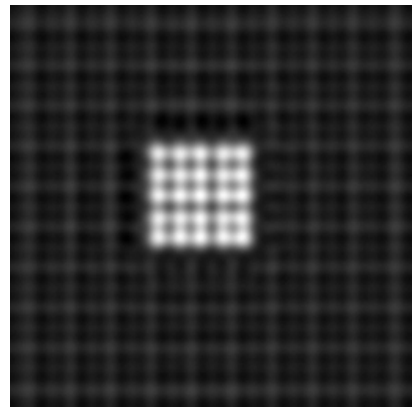

1)

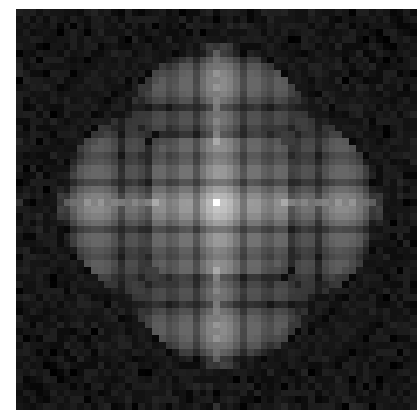

Fig. 1. Digital images of test-objects and amplitudes of their two-dimensional spatial spectra:

a, b, c) test-object, its image formed by the MO, its image after deconvolution, respectively; d, e, f) spatial spectra of (a), (b), (c), respectively; $g, h, i)$ test-object with SMI, its image with SMI, the high resolution digital image, respectively; $j$, $\mathrm{k}$, 1) spatial spectra of $(\mathrm{g}),(\mathrm{h}),(\mathrm{i})$, respectively; 
In the case of the optical microscope with SMI, the spatial harmonics are shifted into the MO bandwidth, they pass the MO can be extracted and shifted back on their original location (see Fig. 1j, k, l). The spatial frequency of SMI is 0.75 of the MO bandwidth. The SMI spatial harmonics can pass through the MO with decreasing its amplitude (see Fig. 1h). It provides higher spatial resolution: in the image made from the optical microscope without SMI, the bars are not resolved (see Fig. 1c), but in the image from the same optical microscope with SMI the bars are clearly resolved (see Fig. 1i).

The minimal resolved spatial period of an imaging channel of an optical microscope with SMI can be calculated using the following formula:

$$
\begin{aligned}
& d_{M}=\frac{1}{v_{O}+v_{M}}=\frac{1}{v_{O} \cdot\left(1+\frac{v_{M}}{v_{O}}\right)}= \\
& =\left(\frac{\lambda}{2 \cdot N A}\right) \cdot \frac{1}{\left(1+\frac{v_{M}}{v_{O}}\right)}=\frac{d}{\left(1+\frac{v_{M}}{v_{O}}\right)}
\end{aligned}
$$

where $v_{\mathrm{O}}$ is the MO bandwidth; $d, d_{M}-$ minimal resolved spatial periods of a grating in optical microscopes without SMI according to Abbe formula and with SMI, respectively; NA - MO numerical aperture; $\lambda$ - operation wavelength.

It is reasonable to consider the formula (5) as a common case of Abbe formula [9]:

$d=\frac{1}{\mathrm{v}_{O}}=\frac{\lambda}{2 \cdot N A}$

The proposed technique has the advantages in comparison with the known techniques [4-8]:

1. It is presented in common analytical form for the two-dimensional digital demodulation. It is applicable for any combination of SMI phases which only should differ. It is very important for practical implementation.

2. It can be applied in the case of low contrast SMI. It is possible due to amplification of SMI amplitudes during digital demodulation.
3. It requires minimal number of calculations to get the high resolution digital images. No iteration procedures are necessary.

4. In the case when $v_{M}<v_{O}$, the bandwidth of an imaging channel in an optical microscope can be expanded up to two MO bandwidths (5) [7].

\section{References}

1. 1. Y. Garini, B.J. Vermolen, I.T. Yong, From micro to nano: recent advances in high-resolution microscopy // Current Opinion in Biotechnology № 6, p. 3-12 (2005).

2. ELYRA Enter the World of Superresolution. Product description. - Jena: Carl Zeiss MicroImaging GmbH, 2009.

3. Applied Precision Delta Vision OMX. 3D-SIM Super-Resolution Imaging. Product description. Issaquah: Applied Precision, Inc., 2009.

4. J.T. Frohn, Super-Resolution Fluorescence Microscopy by Structured Light Illumination. // Dissertation of Doctor of Technical Sciences. Zurich, 2000. - $143 \mathrm{p}$.

5. M. Beck, Extended Resolution in Total Internal Reflection Fluorescence Microscopy. // Dissertation of Doctor of Technical Science. Zurich, 2008. - 120 p.

6. S.S. Hong, Scanning Standing Wave Illumination Microscopy: A Path to Nanometer Resolution in XRay microscopy // Dissertation of Doctor of Philosophy. - Massachusetts, 2005. - 88 p.

7. M.G.L. Gustafsson, L. Shao, P.M. Carlton, R.C.J. Wang, I.N. Golubovskaya, etc. Three-Dimensional Resolution Doubling in Wide-Field Fluorescence Microscopy by Structured Illumination // Biophysical Journal 94, June, p. 4957-4970 (2008).

8. S. Haase, OMX - A Novel High Speed and High Resolution Microscope and its Application to Nuclear Chromosomal Structure Analysis // Dissertation of Doctor of Mathematic - Nature Science. - Berlin, 2005. - 144 p.

9. H.G. Kapitsa, Microscopy from the Very Beginning. $2^{\text {nd }}$ revised edition. - Carl Zeiss: Jena, 1997. 